\title{
Correction to: Attempting to Separate Placebo Effects from Exercise in Chronic Pain: A Systematic Review and Meta-analysis
}

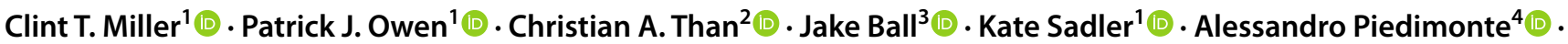 \\ Fabrizio Benedetti ${ }^{4,5}$ - Daniel L. Belavy ${ }^{1,6}(\mathbb{0})$
}

Published online: 19 October 2021

(c) Springer Nature Switzerland AG 2021

Correction to: Sports Medicine

https://doi.org/10.1007/s40279-021-01526-6

\section{Abstract}

\section{Results section, sentence 2:}

In the sentence beginning 'Pairwise meta-analysis...', the value ' $I^{2}=92.4 \%$ ' should have read ' $I^{2}=92.46 \%$ '.

\section{Results section, sentence 3:}

In the sentence beginning 'Exercise training...', the text 'controls $(g[95 \% \mathrm{CI}]: 1.02[0.67,1.36], P<0.001$, $I^{2}=92.99 \%$, studies: $\left.n=42\right)$, usual-care controls ( $g[95 \%$ CI]: 0.65 [0.41, 0.89], $P<0.001, I^{2}=84.82 \%$, studies: $n=33)$ ' should have read ' $(g[95 \% \mathrm{CI}]: 0.99$ [0.66, 1.32], $P<0.001, I^{2}=92.43 \%$, studies: $n=42$ ), usual care controls

The original article can be found online at https://doi.org/10.1007/ s40279-021-01526-6.

Clint T. Miller

c.miller@deakin.edu.au

1 Institute for Physical Activity and Nutrition, School of Exercise and Nutrition Sciences, Deakin University, Geelong, VIC, Australia

2 School of Biomedical Sciences, The University of Queensland, St. Lucia, Brisbane, Australia

3 School of Medicine and Public Health, University of Newcastle, Callaghan, Newcastle, Australia

4 Department of Neuroscience "Rita Levi Montalcini", University of Turin, Turin, Italy

5 Medicine and Physiology of Hypoxia, Plateau Rosà, Zermatt, Switzerland

6 Department of Applied Health Sciences, Division of Physiotherapy, Hochschule für Gesundheit (University of Applied Sciences), Bochum, Germany
( $g$ [95\% CI]: 0.64 [0.44, 0.83], $P<0.001, I^{2}=76.52 \%$, studies: $n=33)^{\prime}$ and when all controls combined ( $g$ [95\% CI]: 0.86 [0.64, 1.07], $P<0.001, I^{2}=91.37 \%$, studies: $\left.n=79\right)$.

\section{Section 3.2, sentence 1:}

In the sentence beginning 'The details of...', the number ' $n=4719$ ' should have read ' $n=4843$ '.

\section{Section 3.4, paragraph 1, sentence 3:}

In the sentence beginning 'Pairwise meta-analysis...', the value ' $I^{2}=92.4 \%$ ' should have read ' $I^{2}=92.46 \%$ ', the value $n=251$ should have read $n=253$.

\section{Section 3.4, paragraph 1, sentence 4:}

In the sentence beginning 'There was no...', the value ' $P=0.249$ ' should have read ' $P=0.250$ '.

\section{Section 3.4, paragraph 2, sentence 1:}

In the sentence beginning 'When pooling all...', the text ' $(g$ [95\% CI]: 0.86 [0.64, 1.07], $P<0.001, I^{2}=91.37 \%$, studies: $n=79$, participants: $n=4,719$; Fig. 4 , Table 2).' should have read ' $\left(g[95 \% \mathrm{CI}]\right.$ : $0.84[0.64,1.04], P<0.001, I^{2}=90.02 \%$, studies: $n=79$, participants: $n=4843$; Fig. 4 , Table 2).'

\section{Section 3.4, paragraph 3 , sentence 1:}

In the sentence beginning 'Sub-group analysis...', the text ' $\left(g[95 \% \mathrm{CI}]: 1.02\right.$ [0.67, 1.36], $P<0.001, I^{2}=92.99 \%$, studies: $n=42$, participants: $n=2,337$; Fig. 5, Table 2);' should have read ' $(g[95 \% \mathrm{CI}]$ : 0.99 [0.66, 1.32], $P<0.001$, $I^{2}=92.43 \%$, studies: $n=42$, participants: $n=2361$; Fig. 5 , Table 2);'. 


\section{Section 3.4, paragraph 4, sentence 1:}

In the sentence beginning, 'When comparing...', the text ' $(g$ [95\% CI]: 0.65 [0.41, 0.89], $P<0.001, I^{2}=84.82 \%$, studies: $n=33$, participants: $n=1,395$; Fig. 6, Table 2),' should read ' $\left(g\right.$ [95\% CI]: 0.64 [0.44, 0.83], $P<0.001, I^{2}=76.52 \%$, studies: $n=33$, participants: $n=2229$; Fig. 6, Table 2),'.

\section{Section 3.5, sentence 2:}

In the sentence beginning 'Among these few...', the value ' $I^{2}=92.4 \%$ ' should have read ' $I^{2}=92.46 \%$ '.

\section{Section 4, paragraph 4, sentence 6:}

In the sentence beginning, 'For pain intensity, the regression coefficient was 0.064 (95\% CI: $-0.002,0.1305 ; P=$ $0.057)$ and $\mathrm{R}^{2}$ was $51.3 \%$ ' should have been ' 0.064 (95\% CI: $-0.002,0.131 ; P=0.057)$ and $\mathrm{R}^{2}$ was $51.3 \%$.

In the sentence beginning, 'The test statistic...', the value ' 14.85 ' should have been ' 14.91 '.

Table 1 should be replaced by the following version: 


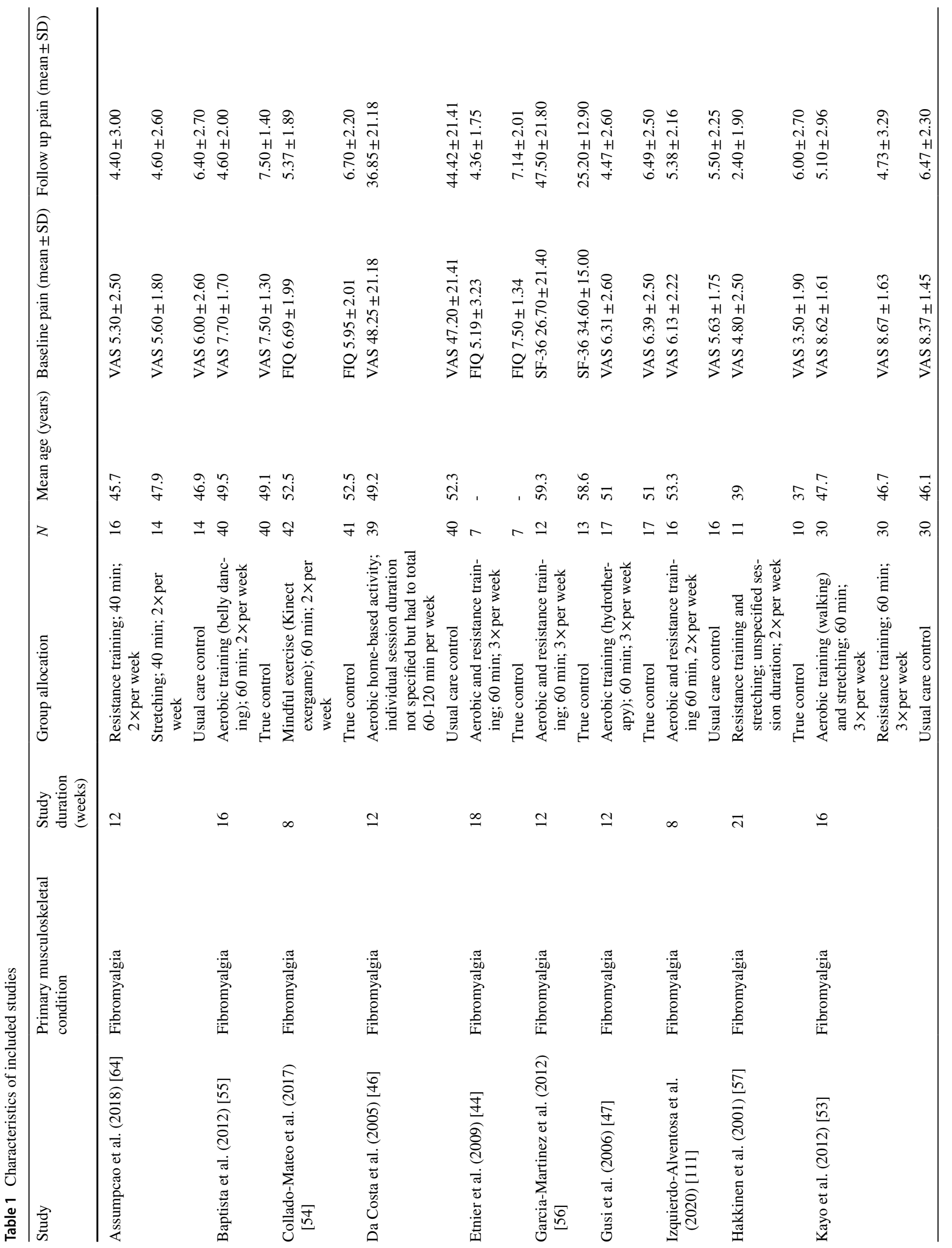




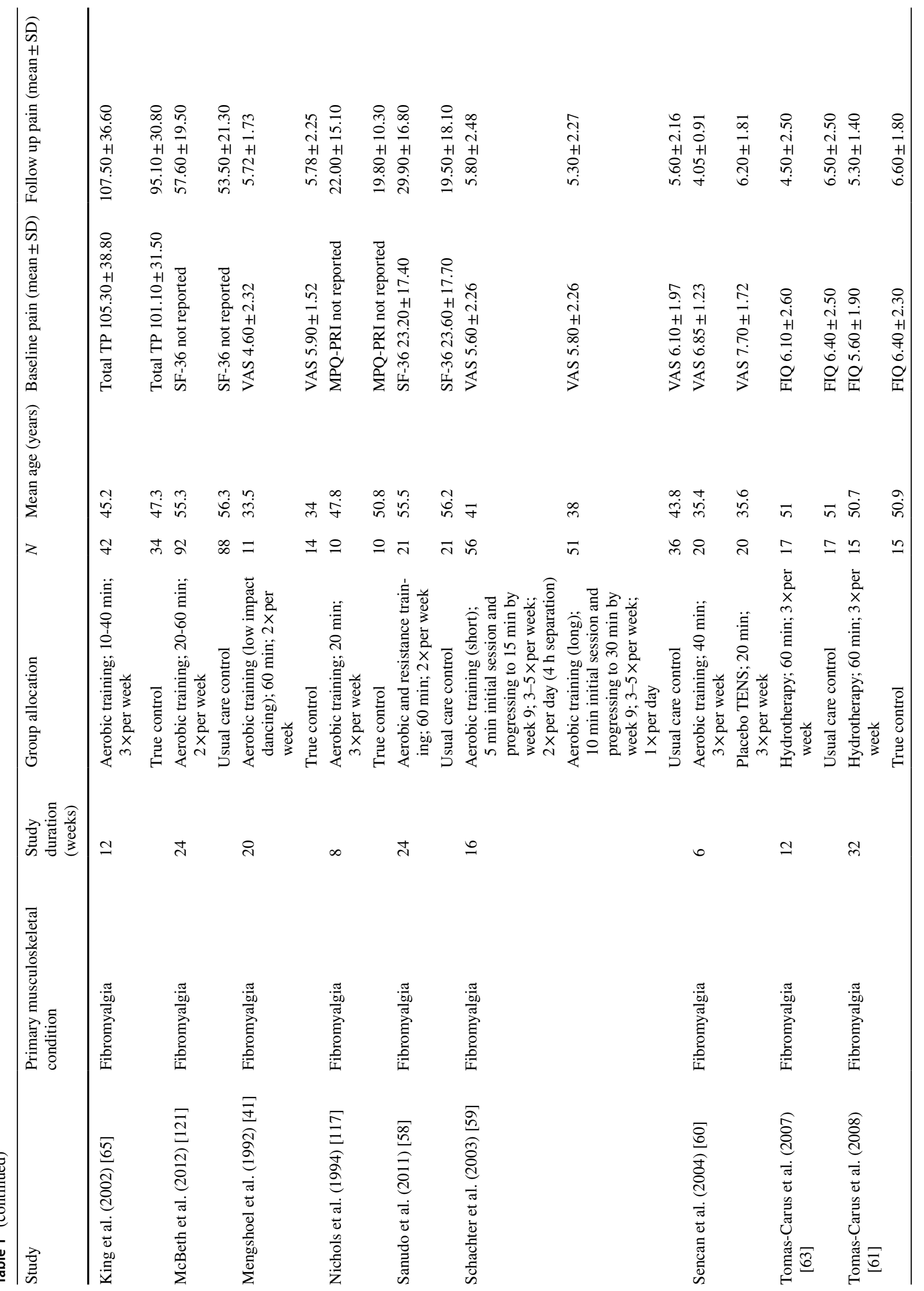




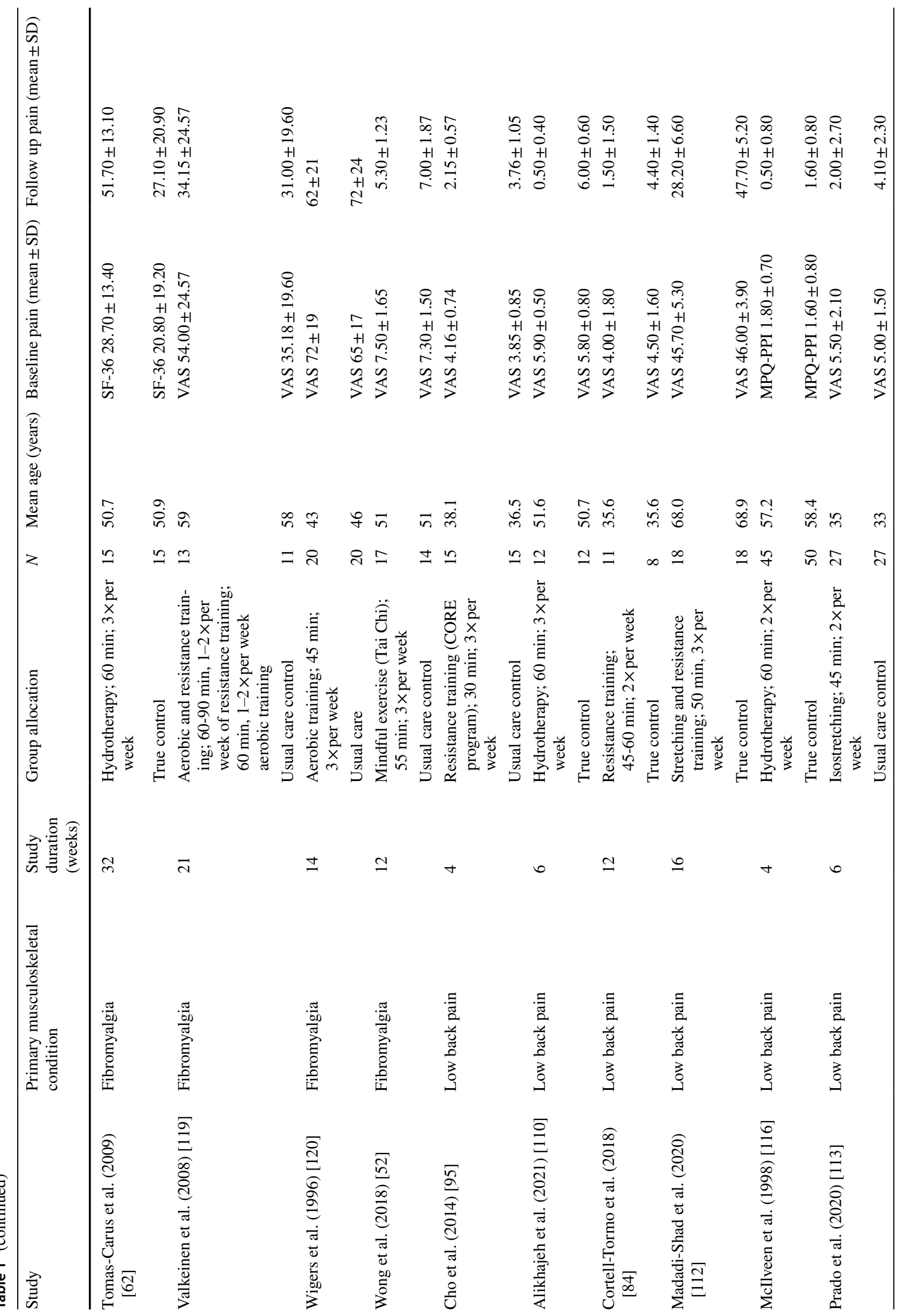




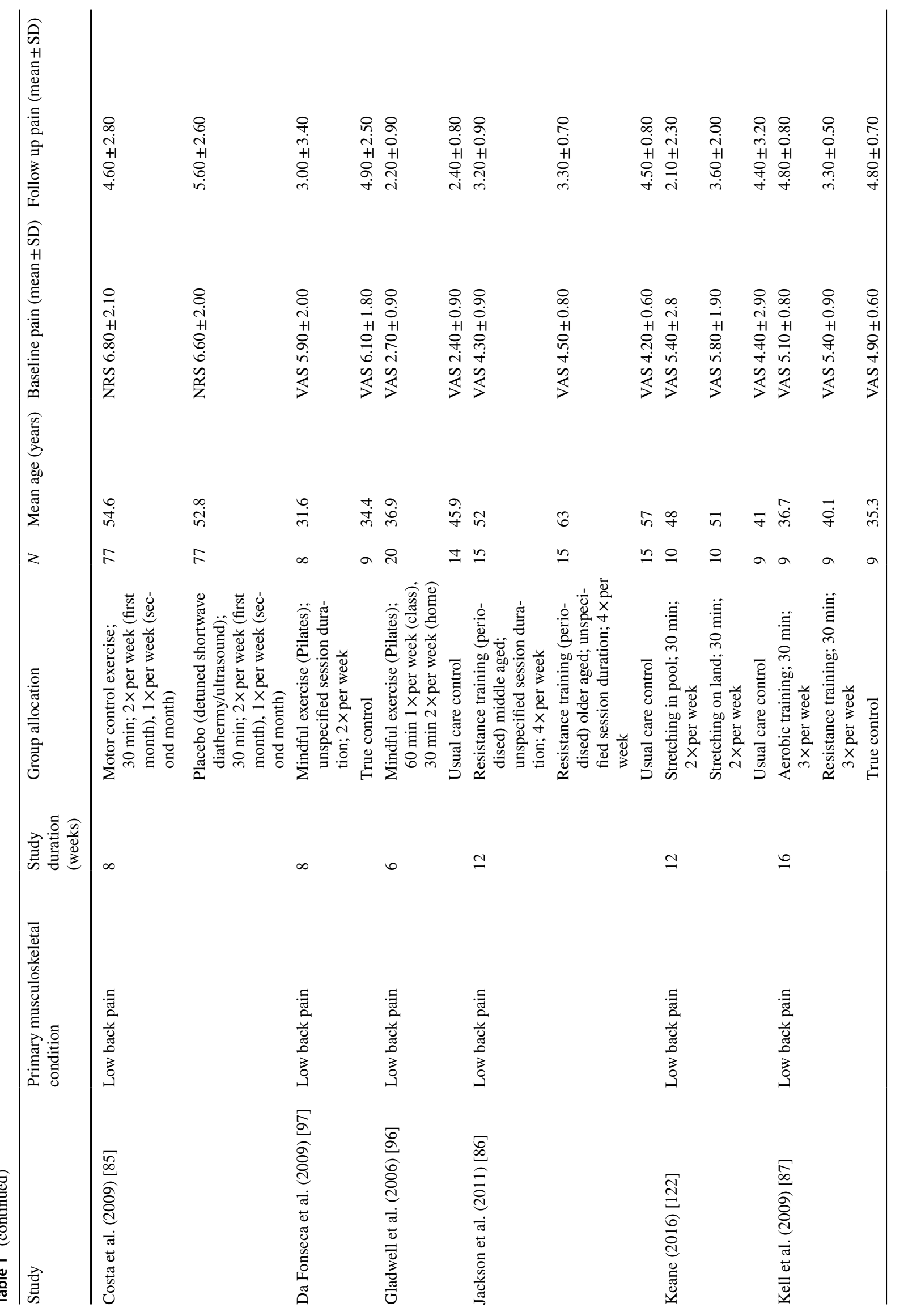




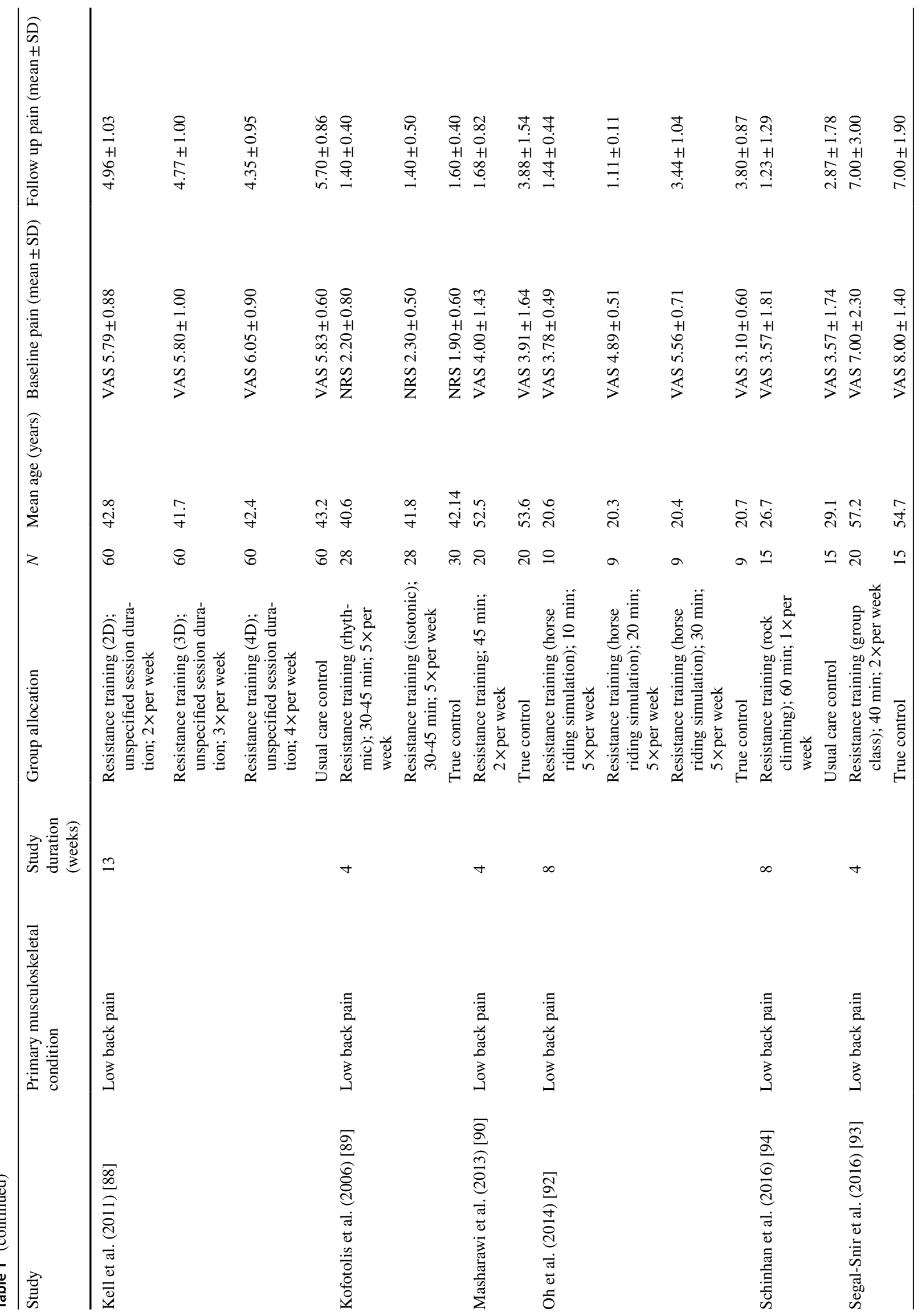




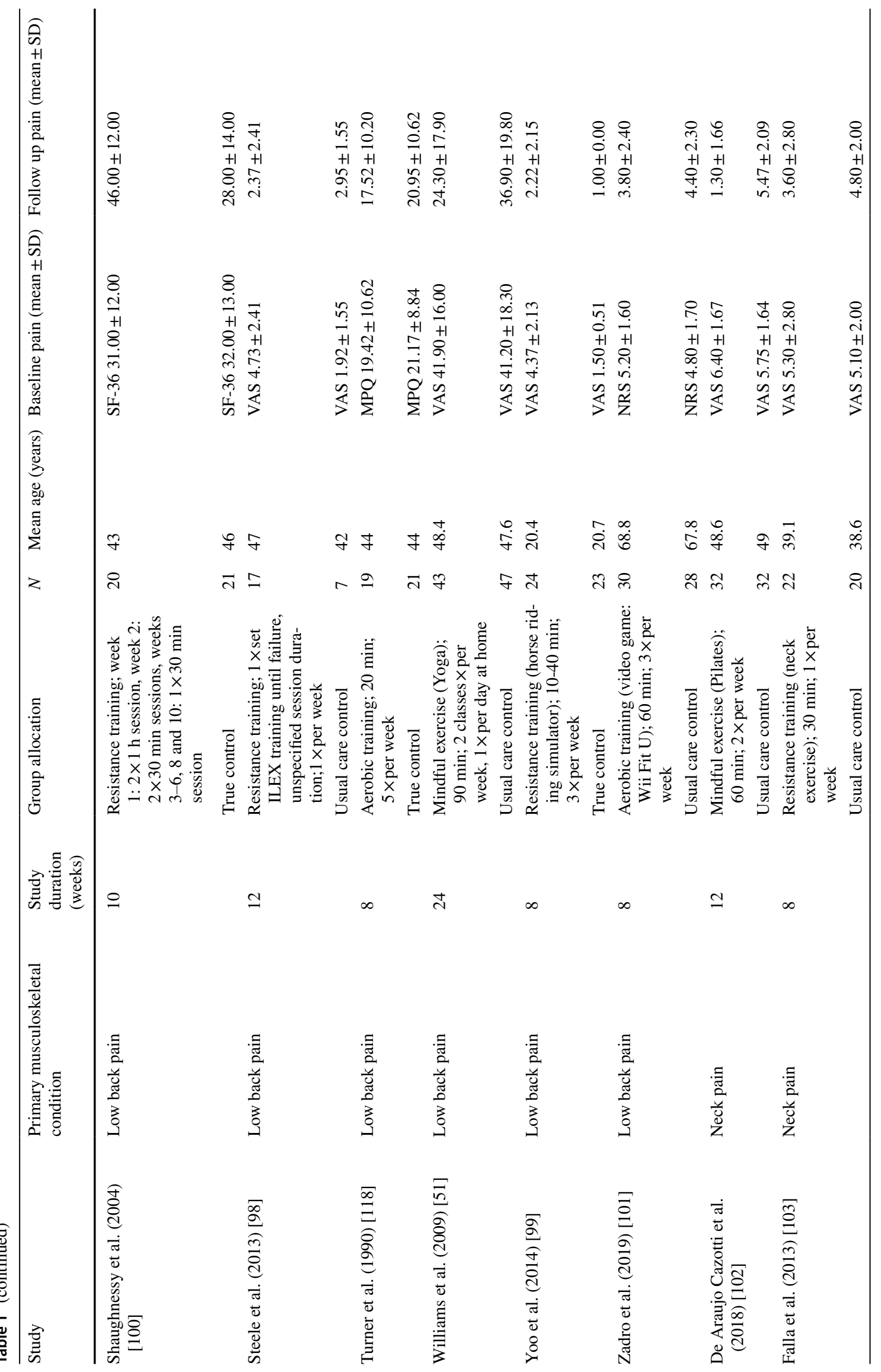




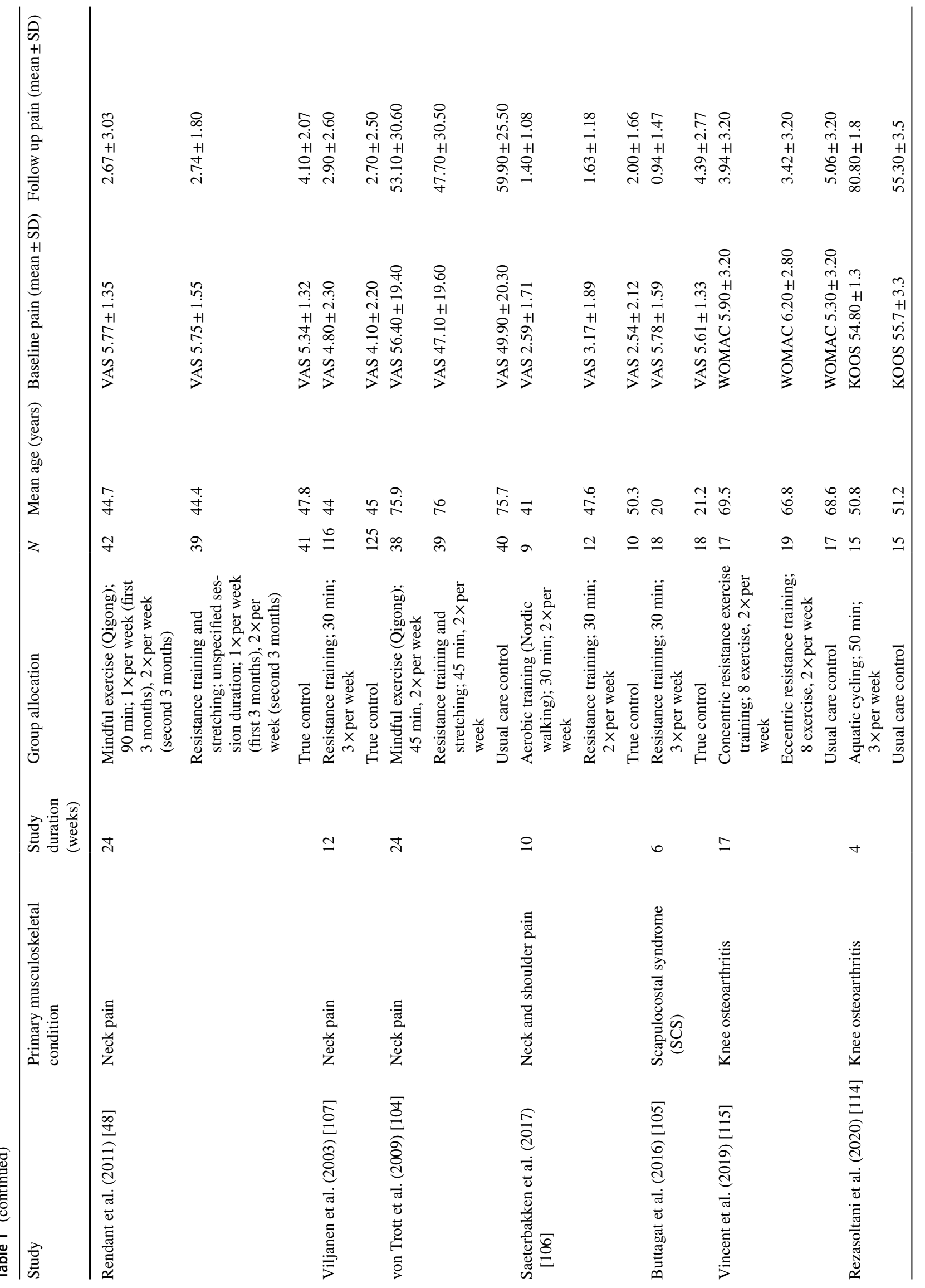




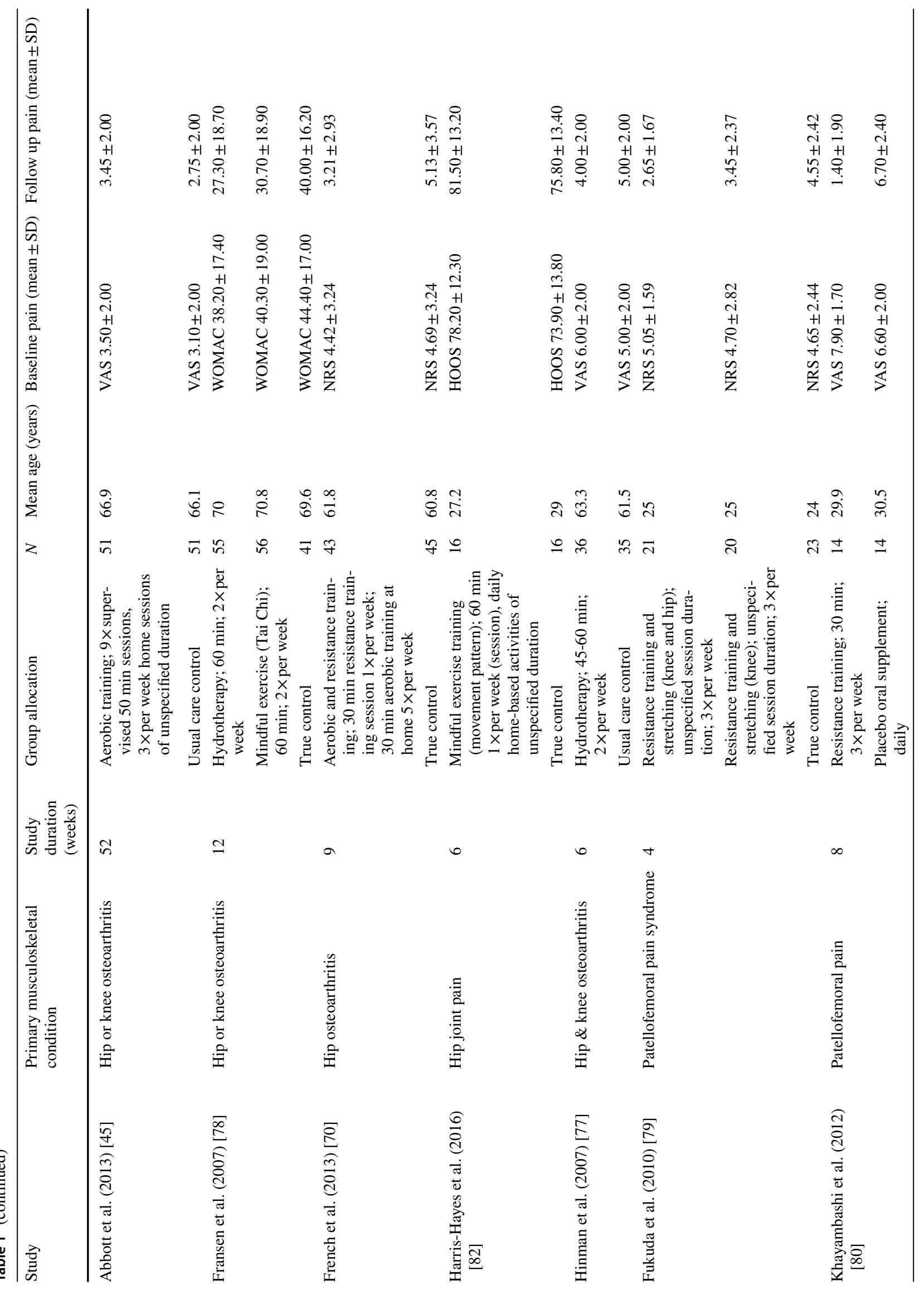




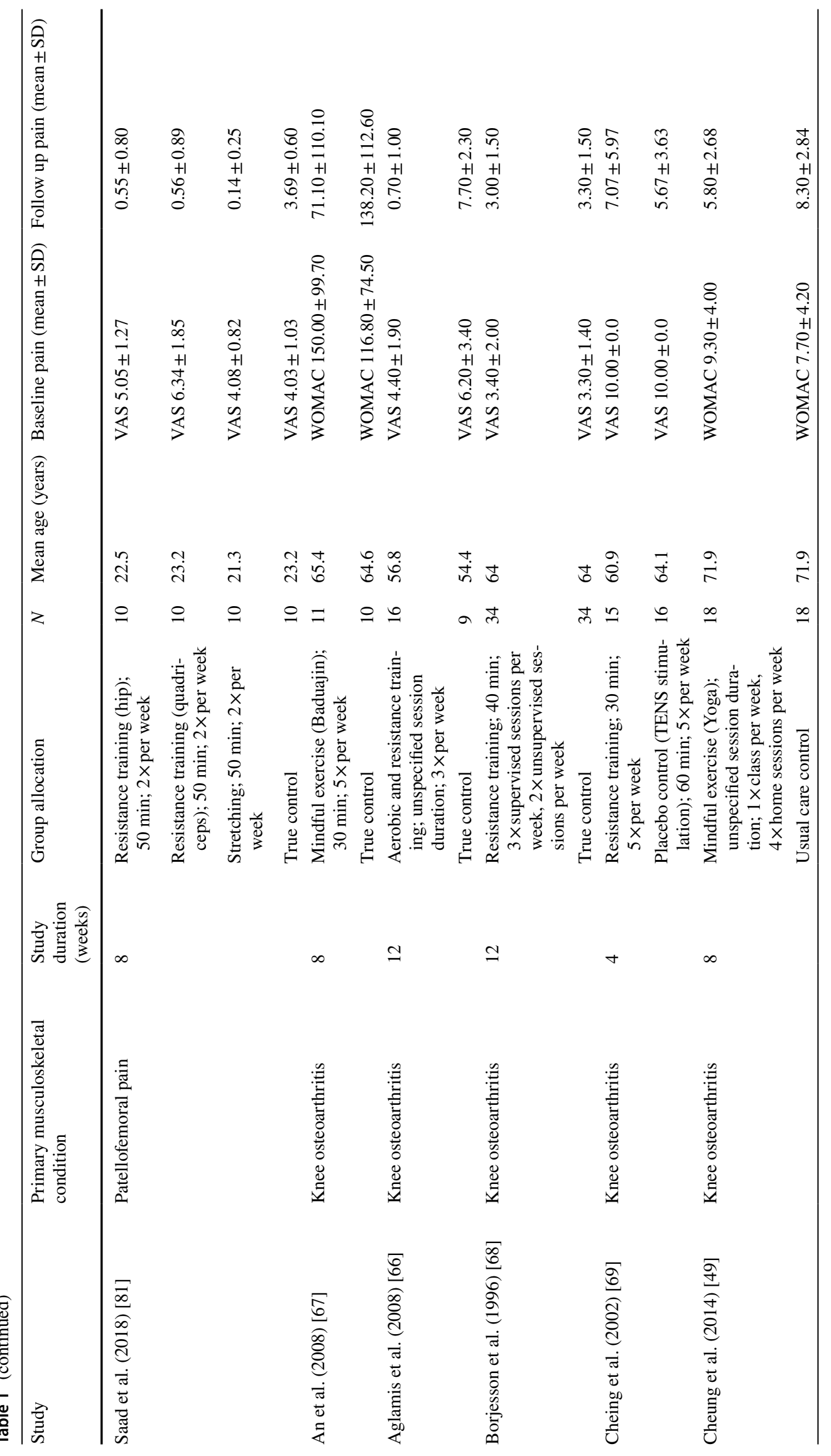




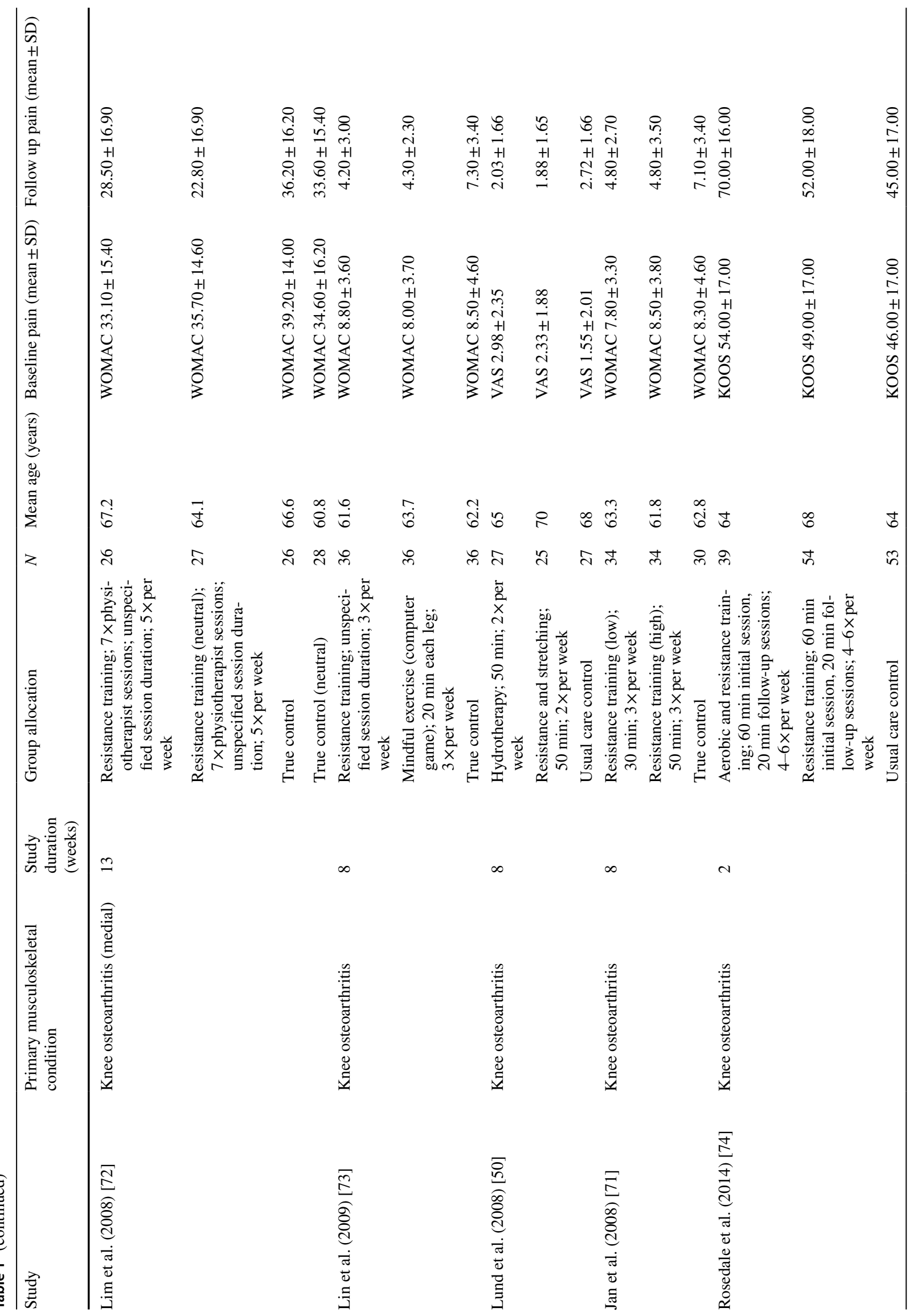




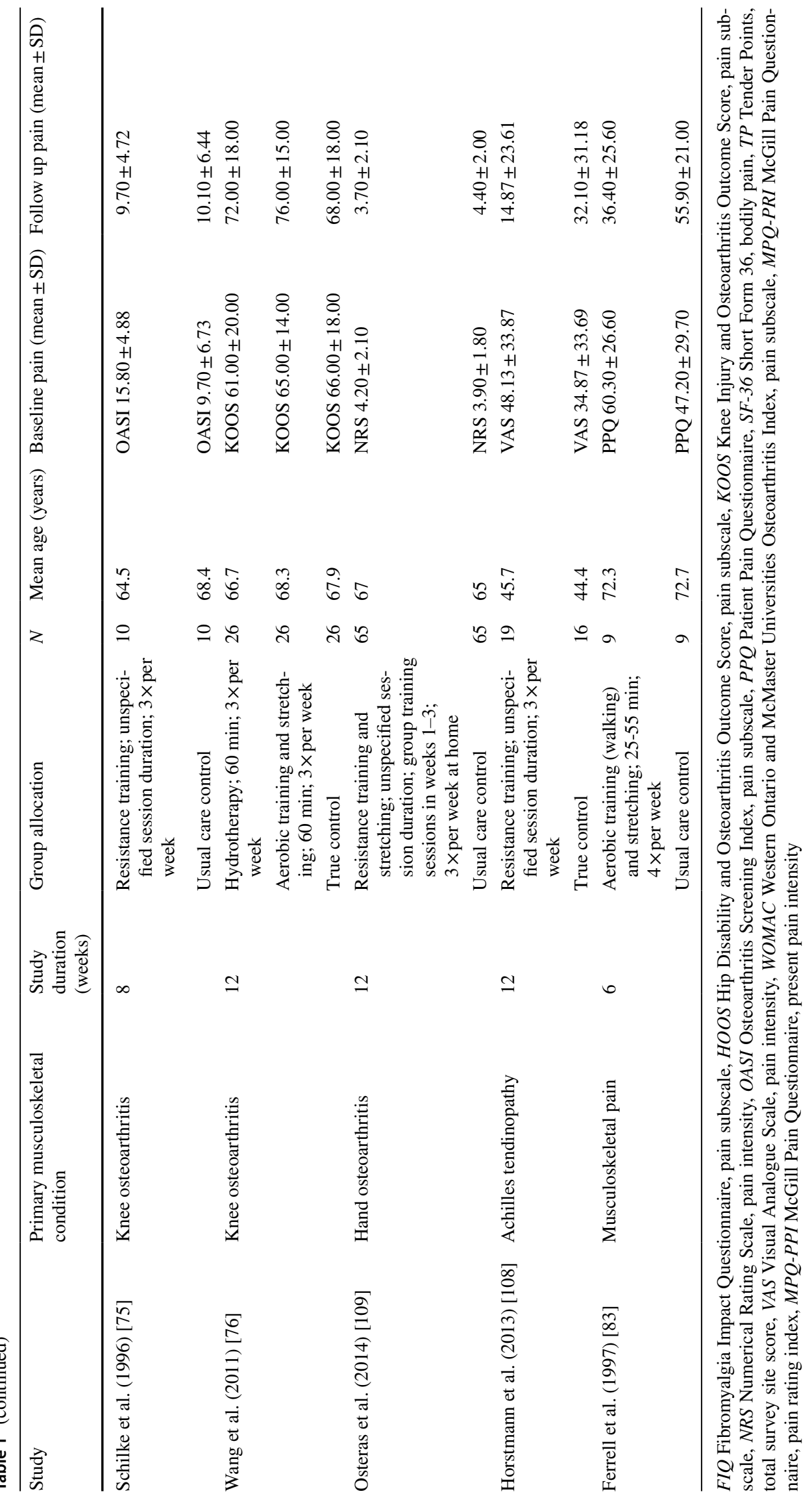


Table 2 should be replaced by the following version:

Table 2 Summary of findings for treatment comparisons

\begin{tabular}{|c|c|c|c|c|c|c|c|c|c|}
\hline Group 1 & Group 2 & Studies & $n$ & Hedges' $g(95 \% \mathrm{CI})$ & $P$ value & $I^{2}(\%)$ & Low ROB (\%) & Egger's P & GRADE* \\
\hline INT: All & CON: Placebo & 4 & 253 & $0.94(-0.17,2.06)$ & 0.098 & $92.46 \%$ & $0 \%$ & 0.250 & Very low $(a, b, c)$ \\
\hline INT: All & CON: All & 79 & 4843 & $0.84(0.64,1.04)$ & $<0.001$ & $90.02 \%$ & $0 \%$ & $<0.001$ & Very low $(a, b, d)$ \\
\hline INT: All & CON: True & 42 & 2361 & $0.99(0.66,1.32)$ & $<0.001$ & $92.43 \%$ & $0 \%$ & $<0.001$ & Very low $(a, b, d)$ \\
\hline INT: All & CON: Usual care & 33 & 2229 & $0.64(0.44,0.83)$ & $<0.001$ & $76.52 \%$ & $0 \%$ & $<0.001$ & Very low $(a, b, d)$ \\
\hline
\end{tabular}

CON control, INT sole exercise training intervention, 95\% CI 95\% confidence interval, ROB risk of bias (percentage of studies with low).

GRADE certainty ratings: very low the true effect is likely markedly different from the estimated effect; low the true effect might be markedly different from the estimated effect; moderate the true effect is likely close to the estimated effect; high the true effect is likely similar to the estimated effect

*a - certainty rated down two grades based on all studies having a high overall risk of bias, b — certainty rated down one grade based on unexplained statistical inconsistency $\left(I^{2} \geq 76.52 \%\right)$, c-certainty rated down one grade based on imprecision due to width of $95 \%$ CI ( $\left.-0.17,2.06\right)$, $\mathrm{d}$ - certainty rated down one grade based on publication bias (Egger's $P<0.05$ ) [40]

Figure 3 should be replaced by the following version:

Figure 4 should be replaced by the following version:

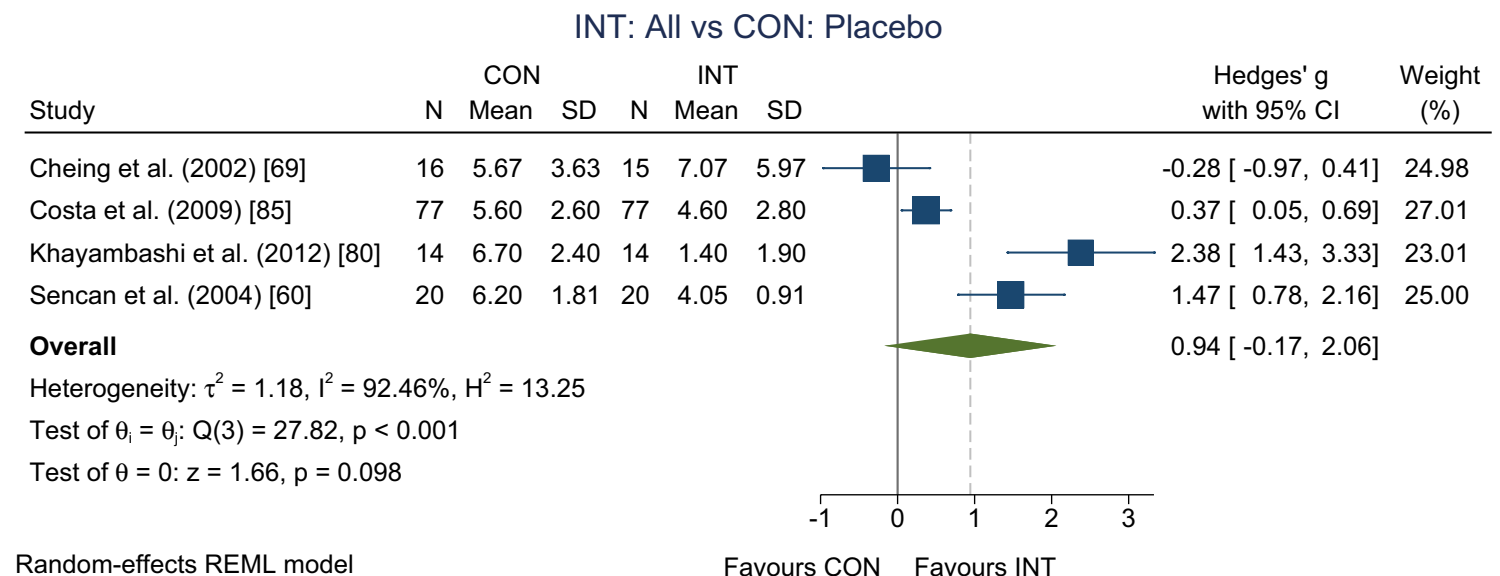

Fig. 3 Forest plot for the meta-analysis investigating the effectiveness of exercise training versus placebo comparators for reducing musculoskeletal pain. Horizontal lines represent standardised mean differ- ence (Hedges' $g$ ) and $95 \%$ confidence intervals. The size of the box represents the weight of each study. The diamond represents the overall estimated effect. INT intervention group, CON control group 
Fig. 4 Forest plot for the metaanalysis investigating the effectiveness of exercise training versus all control comparators for reducing musculoskeletal pain. Horizontal lines represent standardised mean difference (Hedges' $g$ ) and 95\% confidence intervals. The size of the box represents the weight of each study. The diamond represents the overall estimated effect. INT intervention group, CON control group

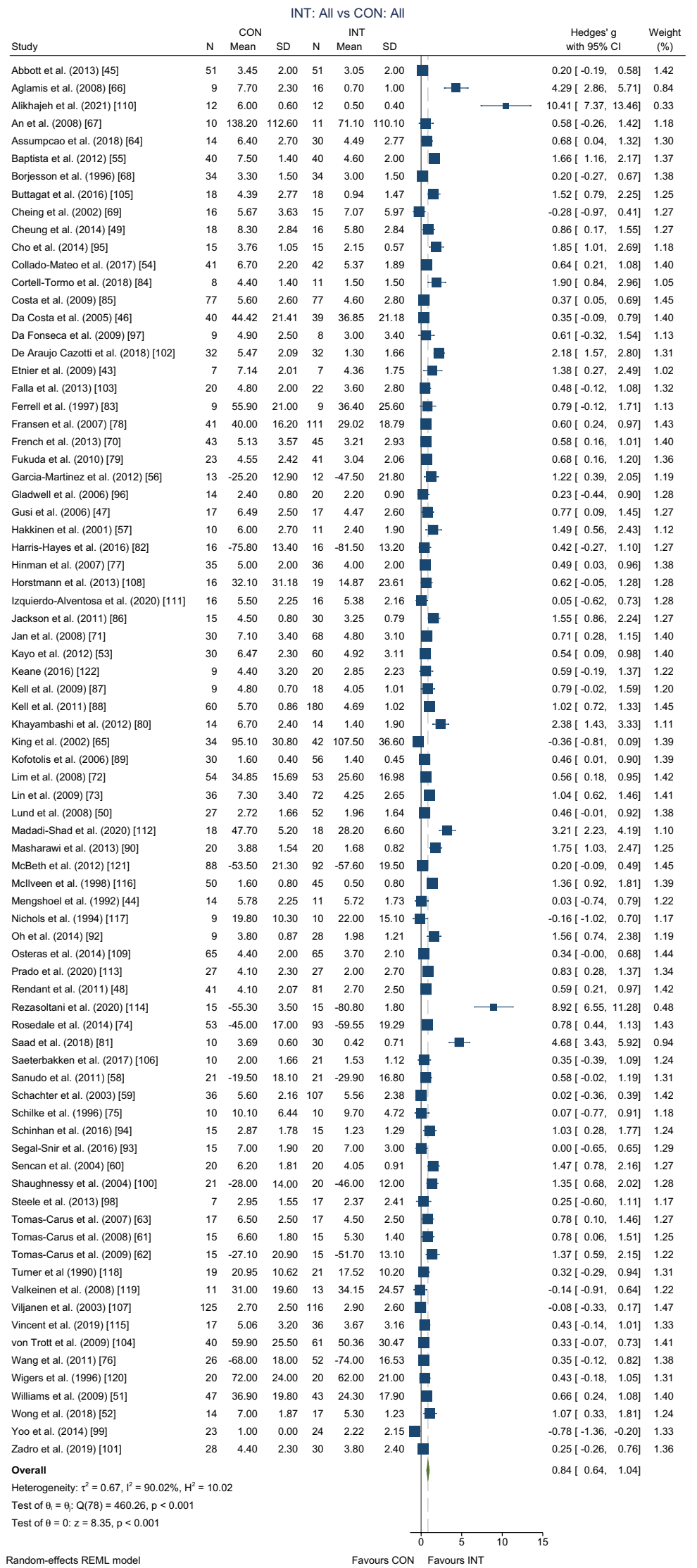


Figure 5 should be replaced by the following version:

Fig. 5 Forest plot for the metaanalysis investigating the effectiveness of exercise training versus true control comparators for reducing musculoskeletal pain. Horizontal lines represent standardised mean difference (Hedges' $g$ ) and 95\% confidence intervals. The size of the box represents the weight of each study. The diamond represents the overall estimated effect. INT intervention group, $C O N$ control group
INT: All vs CON: True

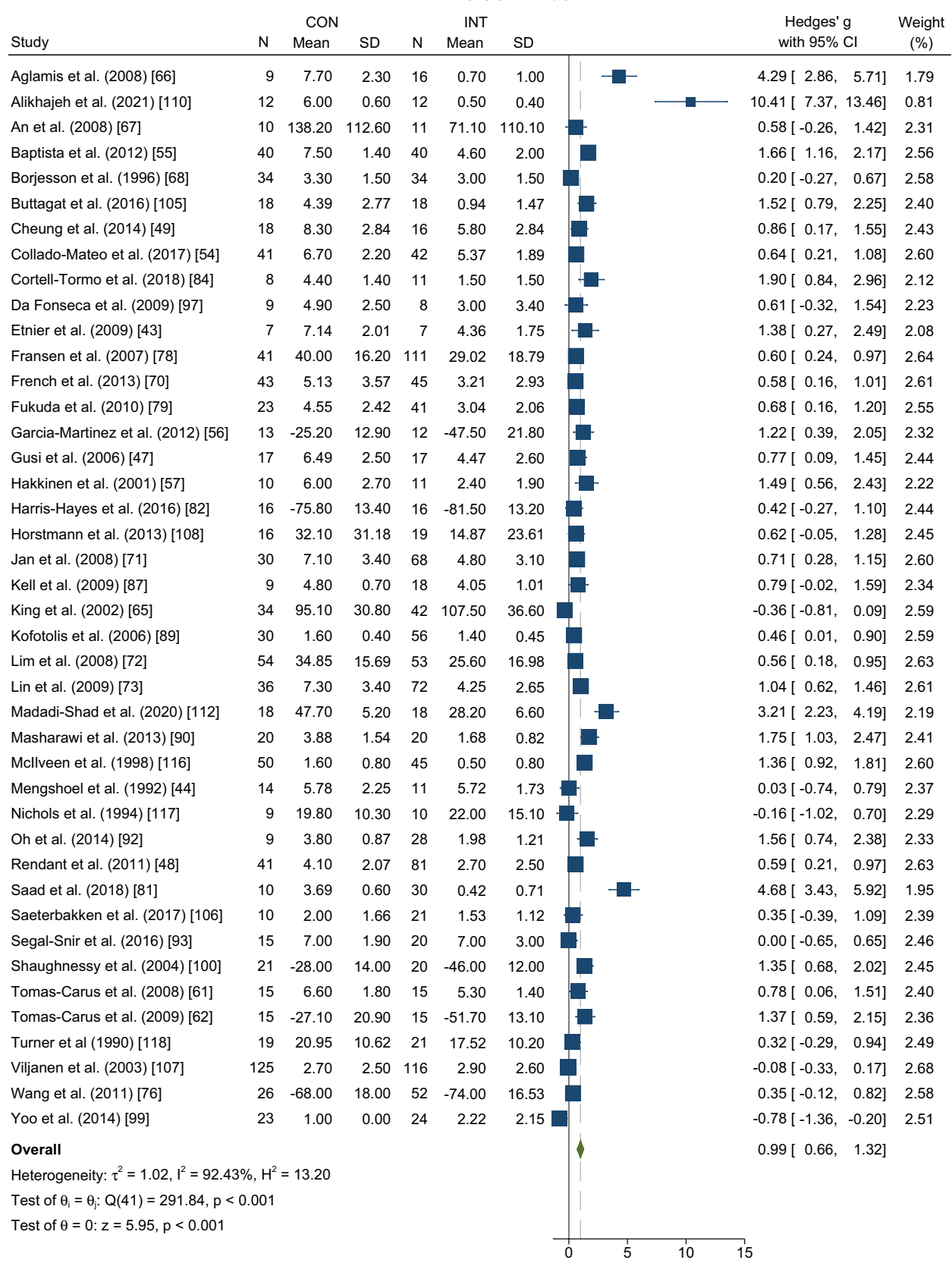

Favours CON Favours INT 
Figure 6 should be replaced by the following version:

Fig. 6 Forest plot for the meta-analysis investigating the effectiveness of exercise training versus usual-care control comparators for reducing musculoskeletal pain. Horizontal lines represent standardised mean difference (Hedges' $g$ ) and $95 \%$ confidence intervals. The size of the box represents the weight of each study. The diamond represents the overall estimated effect. INT intervention group, $C O N$ control group

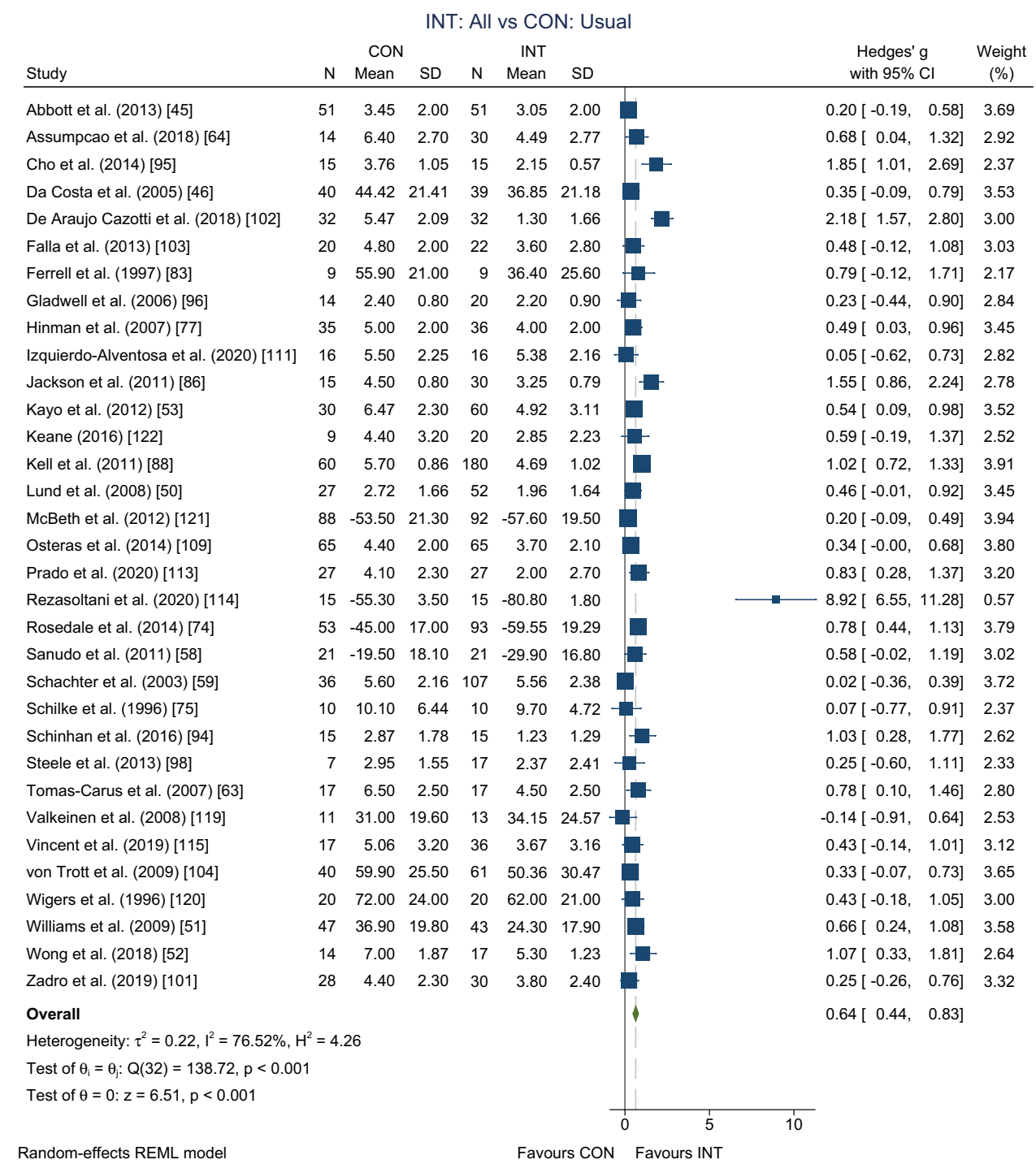

The original article has been updated.

Supplementary Information The online version contains supplementary material available at https://doi.org/10.1007/s40279-021-01578-8. 